\title{
¿QUÉ PROCESOS MATEMÁTICOS SE MOVILIZAN CUANDO SE ENSEÑA PROBABILIDAD? UN ESTUDIO DE CASO EN EL AULA DE EDUCACIÓN INFANTIL
}

\author{
WHAT MATHEMATICAL PROCESSES ARE MOBILIZED WHEN \\ PROBABILITY IS TAUGHT? A CASE STUDY IN THE EARLY CHILDHOOD \\ EDUCATION CLASSROOM
}

\author{
Claudia Vásquez \\ Pontificia Universidad Católica de Chile - Chile \\ cavasque@uc.cl \\ Nataly Pincheira \\ Universidad de Girona - España \\ nataly.pincheira@udg.edu
}

\begin{abstract}
Resumen
En este trabajo se presenta un análisis de los procesos matemáticos que gestiona un profesor en el aula de Educación Infantil al enseñar probabilidad. Se ha realizado un estudio exploratorio con un enfoque cualitativo, utilizando como diseño de investigación el estudio de caso. La técnica de recolección de datos obedece a la observación no participante a través de la videograbación de dos sesiones de clase. Los resultados muestran la presencia de los procesos matemáticos de resolución de problemas, razonamiento y prueba, comunicación, conexiones y representación con distintos niveles de profundidad y atención en la sesión de clase analizada. Se concluye que la gestión de las experiencias de aprendizaje que realiza el profesorado constituye un aspecto clave en la movilización de los procesos matemáticos, provocando que emerjan procesos que no son del todo evidente en el diseño de tales experiencias. La formulación de buenas preguntas durante la gestión de las experiencias de aprendizaje visibiliza los procesos matemáticos e invita a los niños a razonar, comunicar, argumentar y justificar sus acciones e ideas matemáticas en torno a la probabilidad.

Palabras chave: procesos matemáticos, probabilidad, enseñanza, Educación Infantil.
\end{abstract}

\begin{abstract}
This paper presents an analysis of the mathematical processes managed by a teacher in the Early Childhood Education classroom when teaching probability. An exploratory study has been carried out with a qualitative approach, using a case study research design. The data collection technique is based on non-participant observation through the video recording of two classroom sessions. The results show the presence of the mathematical processes of problem solving, reasoning and proof, communication, connections and representation with different levels of depth and attention in the classroom session analysed. It is concluded that teacher management of learning experiences is a
\end{abstract}


key aspect in the mobilization of mathematical processes, causing processes to emerge that are not entirely evident in the design of such experiences. The formulation of good questions during the management of learning experiences makes mathematical processes visible and invites children to reason, communicate, argue and justify their mathematical actions and ideas around probability.

Keywords: mathematical processes, probability, teaching, Early Childhood Education.

\section{INTRODUCCIÓN}

En el informe El Futuro de la Educación y las Competencias 2030 (OCDE, 2019), se destaca la importancia de avanzar en el desarrollo de habilidades y conocimientos que permitan formar ciudadanos capaces de "extraer información significativa de los datos, comprender qué significan los datos, incluyendo cómo leerlos de manera apropiada, extraer conclusiones, así como reconocer cuándo se utilizan de manera engañosa o inapropiada" (p.5). Para ello, es imperativo comenzar el inicio del estudio de la estadística y la probabilidad desde temprana edad, pues las niñas y los niños desde pequeños desarrollan su comprensión del mundo por medio de un razonamiento causal y estadístico distinguiendo entre aquello que es probable y aquello que no lo es- como parte del desarrollo de sus conocimientos científicos, matemáticos y sociales (YUROVSKY; BOYER; SMITH; YU, 2013). Pues, no cabe duda de que los conocimientos matemáticos que adquieran las niñas y los niños durante sus primeros años de escolaridad serán clave para su aprendizaje y desempeño posterior sobre todo en temas vinculados a matemática (PASSOLUNGHI; LANFRANCHI, 2012). Por tanto, y de acuerdo con el Consejo Nacional de Profesores de Matemáticas de Estados Unidos (NCTM, por sus siglas en inglés), es necesario desarrollar una sólida base matemática en los primeros años, puesto que es en estas edades que los niños construyen sus creencias, sobre qué son las matemáticas, sobre qué significa saber y utilizar matemáticas, y sobre sí mismos como aprendices de matemáticas (NCTM, 2000). Asimismo, la Asociación Australiana de Profesores de Matemáticas y Primera Infancia en Australia (2012) sostiene que "todos los niños, en sus primeros años, son capaces de acceder a grandes ideas matemáticas, relevantes para su vida actual y, a su vez, fundamentales para su futuro aprendizaje de las matemáticas y para otros aprendizajes" (p. 2). Por tanto, es necesario brindar a las niñas y los niños de Educación Infantil, experiencias de aprendizaje de calidad. En este sentido, la National Association for the Education of Young Children y el NCTM, señalan un conjunto de recomendaciones a considerar por el profesorado en sus prácticas de aula con el fin de 
promover una Educación Matemática de calidad, como por ejemplo, "la importancia de utilizar currículos y prácticas docentes que fortalezcan los procesos infantiles de resolución de problemas y razonamiento, así como los de representación, comunicación y conexión de ideas matemáticas" (NAEYC; NCTM, 2013, p.7). En vista de ello, los procesos matemáticos constituyen una de las características mas importantes para definir actividades matemáticamente competenciales (NCTM, 2000). No obstante, a pesar de las diversas recomendaciones respecto a la enseñanza y aprendizaje de la matemática en estas edades, existe todavía poca tradición en el caso particular de la enseñanza de la estadística y la probabilidad en los niveles de Educación Infantil y los primeros años de Educación Primaria (VÁSQUEZ; CORONATA; RIVAS, en prensa), pese a que el profesorado de estos niveles educativos reconoce el valor, utilidad e importancia de enseñar estos temas desde las primeras edades (VÁSQUEZ, ALVARADO; RUZ, 2019). Si bien es cierto, los currículos de Educación Matemática de infantil y primaria de numerosos países han incluido explícitamente el estudio de la estadística y la probabilidad desde temprana edad (VÁSQUEZ; CABRERA, en revisión), aun son escasos los estudios referidos a la implementación de experiencias de aprendizaje sobre estos temas en las primeras edades (VÁSQUEZ; ALSINA, 2019). En concreto, en trabajos preliminares (e.g. ALSINA, 2013, 2017; VÁSQUEZ; ALSINA, 2017, 2019; VÁSQUEZ; DÍAZ-LEVICOY, CORONATA; ALSINA, 2018), se plantean numerosas propuestas para abordar la enseñanza de la estadística y probabilidad en edades tempranas, asimismo sobre cómo se aborda su enseñanza en los libros de texto de infantil (VÁSQUEZ; DÍAZ-LEVICOY; ARTEAGA, 2020; VÁSQUEZ; CORONATA; RIVAS, en prensa). No obstante, poco se conoce sobre los conocimientos que pone en juego el profesorado al enseñar; sobre cómo el profesorado promueve el aprendizaje de la matemática en general, y de la estadística y la probabilidad en particular en esta etapa educativa. Dichos conocimientos abarcan un amplio conjunto de capacidades cognitivas y de habilidad para resolver situaciones en el aula, tales como el conocimiento de la disciplina, el conocimiento pedagógico y el conocimiento didáctico del contenido, junto con habilidades de percepción, interpretación y diagnóstico; lo que implica que "el profesorado de esta etapa educativa pueda integrar el trabajo sistemático de los procesos matemáticos en sus prácticas docentes, y poder avanzar así en el logro de una sociedad que tenga la capacidad de pensar y razonar matemáticamente, y una base útil 
de conocimientos y destrezas matemáticas” (ALSINA, 2014, p.7).

En este estudio hemos querido dar un paso más, y focalizarnos en el análisis del conocimiento profesional del profesorado de Educación Infantil para enseñar probabilidad, más especificamente en los procesos matemáticos. Por consiguiente, en este trabajo nos enfocamos en las preguntas que realiza el profesor y en cómo guía el aprendizaje de la probabilidad, con el propósito de describir cómo el profesor gestiona los procesos matemáticos en el aula de Educación Infantil al enseñar probabilidad. Nos centramos en los procesos matemáticos, puesto que son la clave para promover competencia matemática, es decir, para "gestionar el conocimiento, las habilidades y las emociones para conseguir un objetivo a menudo más cercano a situaciones funcionales y en contextos de vida cotidiana que a su uso académico" (ALSINA, 2019, p. 17).

\section{LOS PROCESOS MATEMÁTICOS EN EDUCACIÓN INFANTIL}

Los procesos matemáticos ofrecen un conjunto de herramientas que facilitan la adquisición y uso de los contenidos que se abordan en el currículo de matemática, pues a partir de ellos las niñas y los niños se introducen en las formas de pensar propias de las matemáticas como: razonar, argumentar, descubrir, representar, modelizar, etc. Estos procesos permitirán construir nuevos conocimientos $\mathrm{y}$, especialmente, otorgar aplicabilidad a los contenidos tratados, vinculándoles no solo con otros contenidos matemáticos y de otras disciplinas, sino también con problemáticas provenientes de diversos contextos (ALSINA, 2014).

En el caso del bloque de contenidos de análisis de datos y probabilidad, que es el que nos ocupa en este estudio, se observa que este se aborda con un gran énfasis en los primeros años de edad y de manera continua a lo largo de las distintas etapas educativas. Esto favorecerá que las niñas y los niños profundicen progresivamente en las ideas y conceptos clave de estadística y probabilidad, a través de experiencias diversas que conectan tales conocimientos con una variedad de escenarios: la escuela, el hogar, la vida diaria, etc. Esto implica que las niñas y los niños desarrollen un pensamiento crítico desde temprana edad, de manera que lleguen a ser ciudadanos y consumidores bien informados, capaces de tomar decisiones a partir del análisis de datos y probabilidad (VÁSQUEZ, 2018, 2020; VÁSQUEZ; CORONATA; RIVAS, en prensa). Para esto, es fundamental poner 
énfasis en el desarrollo de habilidades, conceptos y procedimientos cada vez más complejos, que constituyan un progreso a través de los diferentes niveles educativos. De manera tal que, al finalizar la etapa escolar los estudiantes cuenten con un conocimiento sólido de estadística y probabilidad, que les permita establecer conexiones con otras disciplinas, así como su aplicación a la vida diaria. Es así como, desde este enfoque, respecto al tema de estadística para el nivel Pre-K2 se enfatiza en la necesidad de que los estudiantes propongan cuestiones para investigar, organizar las respuestas y crear representaciones para sus datos. En cuanto a la probabilidad en esta etapa educativa, esta se debe abordar a un nivel informal donde los estudiantes analicen situaciones de incertidumbre cercanas a su realidad, emitan juicios fundamentados utilizando lenguaje asociado a lo cotidiano para fundar e introducir poco a poco un lenguaje probabilístico, para designar la posibilidad de ocurrencia de un determinado suceso (imposible, probable, seguro, etc.). Este tipo de lenguaje sin duda "constituye un elemento clave, sobre todo en los primeros niveles educativos, para incorporar progresivamente un lenguaje probabilístico y avanzar así en la construcción del conocimiento sobre probabilidad" (VÁSQUEZ; ALSINA, 2017, p. 457).

Por consiguiente, es fundamental que en la Educación Infantil la estadística y la probabilidad se aborde a partir de situaciones que se encuentran en la vida cotidiana para así favorecer su observación, comprensión e interpretación. Para ello, es primordial abordar la enseñanza de estos temas desde lo cotidiano, desde una articulación entre contenidos y procesos matemáticos, para así garantizar el aprendizaje de los conocimientos necesarios que permitan a los estudiantes constituirse como ciudadanos matemáticamente competentes. Desde este prisma, a continuación, se describen aspectos centrales vinculados a cada proceso matemático entre los 4 a los 8 años.

\section{La resolución de problemas}

La resolución de problemas se presenta de manera natural producto de la curiosidad innata, inteligencia y flexibilidad al enfrentar situaciones nuevas. Es por ello, que entre los 4-8 años se debería animar a los estudiantes a resolver situaciones que provengan de contextos diversos, que vayan desde rutinas diarias a situaciones matemáticas que surgen de juegos y cuentos, que les brinde oportunidades -según sus diferentes grados de conocimientos y destrezas- de usar, ampliar y construir nuevos conocimientos matemáticos. 
En este sentido, la NCTM (2000) recomienda iniciar a las niñas y los niños desde temprana edad en: construir nuevos conocimientos matemáticos a través de la resolución de problemas; resolver problemas que surjan de las matemáticas y de otros contextos; aplicar y adaptar una variedad de estrategias para resolver problemas; controlar el proceso de resolución de problemas matemáticos y reflexionar sobre él.

\section{El razonamiento y prueba}

Las niñas y los niños desde pequeños comienzan a desarrollar su razonamiento lógico, siendo este modificado constantemente producto de las experiencias. Así poco a poco, a partir de actividades de clasificación, ordenación, relación y comparación que los llevan al reconocimiento de patrones, van desarrollando su razonamiento matemático. En esta línea el NCTM (2000), propone una enseñanza que estimule a los estudiantes desde la Educación Infantil y a diferentes grados según la edad en: reconocer el razonamiento y prueba como aspectos fundamentales de las matemáticas; formular e investigar conjeturas matemáticas; desarrollar y evaluar argumentos y demostraciones matemáticas; y elegir y utilizar varios tipos de razonamiento y métodos de prueba. Para ello, es esencial el planteamiento de buenas preguntas que conduzcan, a partir del uso de ejemplos y contraejemplos, a generalizar y comprobar tales generalizaciones (CARPENTER; LEVI, 1999).

\section{La comunicación}

La comunicación es un elemento clave a considerar de manera sistemática en la enseñanza y aprendizaje de la matemática, pues permite articular, organizar y consolidar el pensamiento matemático. Esta comunicación matemática comienza a manifestarse desde muy temprano (primeros meses de vida). Por tanto, ya en la etapa de Educación Infantil es necesario comenzar a ayudar a las niñas y los niños que se expresen sobre matemáticas, que reflexionen sobre sus conocimientos y formas de resolver problemas. Para ello, el NCTM (2000), propone una enseñanza centrada en: la organización y consolidación del pensamiento matemático a través de la comunicación; comunicación del pensamiento matemático con coherencia y claridad a los compañeros, profesores y otras personas; análisis y evaluación de las estrategias y pensamiento matemático de los demás; y uso de lenguaje matemático para expresar ideas matemáticas con precisión. 


\section{Las conexiones}

En lo que respecta al aprendizaje de las matemáticas, este surge a partir de las conexiones entre las ideas matemáticas intuitivas e informales que los estudiantes poseen desde pequeños. Por consiguiente, el proceso de enseñanza y aprendizaje en infantil debe conectar lo que las niñas y los niños aprenden en la escuela con tales ideas intuitivas e informales. De acuerdo con Alsina (2014), es posible distinguir tres tipos de conexiones en la Educación Infantil, aquellas relacionadas con las conexiones entre los diferentes bloques de contenido matemático y entre contenidos y procesos matemáticos (conexiones intradisciplinares); conexiones con otras áreas de conocimiento (conexiones interdisciplinares); y conexiones con el entorno (enfoque globalizado). En esta dirección, y de acuerdo con el NCTM (2000), es importante un enfoque de enseñanza que favorezca un trabajo progresivo en torno a: reconocer y usar conexiones entre ideas matemáticas; comprender cómo las ideas matemáticas se interconectan y construyen unas sobre otras para producir un todo coherente; por último, reconocer y aplicar las matemáticas en contextos no matemáticos.

\section{La representación}

Las representaciones a través de objetos físicos, el lenguaje natural, gestos, dibujos, diagramas y símbolos inventados o convencionales; permiten acceder a la comprensión de las ideas matemáticas de los estudiantes. De acuerdo con Alsina (2014), las representaciones son "un proceso indispensable para poder aprender. Si no hay representación del conocimiento no hay aprendizaje" (p. 16). Desde este prisma, el NCTM (2000) plantea que desde temprana edad se debe promover de manera progresiva una enseñanza centrada en: crear y usar representaciones para organizar, registrar, y comunicar ideas matemáticas; seleccionar, aplicar y traducir representaciones matemáticas para resolver problemas; y usar representaciones para modelizar e interpretar fenómenos físicos, sociales y matemáticos.

\section{METODOLOGÍA}

Dado que el propósito de este estudio es describir cómo el profesor gestiona los procesos matemáticos en el aula de Educación Infantil al enseñar probabilidad, es que hemos optado por realizar un estudio exploratorio bajo un enfoque de investigación cualitativo (HERNÁNDEZ; FERNÁNDEZ; BAPTISTA, 2010), utilizando como diseño 
de investigación el estudio de caso (STAKE, 2020). Pues a través de la investigación no participante se indaga en las características de un proceso de enseñanza (COHEN; MANION; MORRISON, 2018).

\section{Contexto y participantes}

El estudio se llevo a cabo con 12 niñas y 11 niños de Educación Infantil cuyas edades fluctúan entre los 4 a 6 años, y no han recibido instrucción previa vinculada a probabilidad, saben contar (reconocer cantidades elementales de un conjunto de elementos) hasta 20, además manejan algunas relaciones de orden de cantidad con ayuda del profesor. El profesor a cargo de este grupo cuenta con especialidad para la enseñanza de las matemáticas en estas edades, y cuenta con 6 años de experiencia en el aula de infantil. Para seleccionar al profesor participante del estudio, se consideraron los criterios o características de experto, de acuerdo con el planteamiento de Rojas, Carrillo y Flores (2012): a) realizar clases de matemática en el nivel de Educación Infantil con una experiencia de al menos 5 años; b) ser reconocido y recomendado por sus compañeros y directivos, por sus buenas prácticas de enseñanza en matemática; c) participar de manera constante en actividades de actualización en relación con la matemática y su enseñanza; d) haber enseñando temas vinculados a probabilidad, más de una vez, en los últimos 3 años; y e) haber logrado un buen desempeño de sus estudiantes, en matemática, en mediciones locales y nacionales. De este modo, el estudiar y analizar las prácticas de enseñanza de profesores con características de expertos aportará información respecto del conocimiento profesional para la enseñanza de la probabilidad en el aula de infantil, pues de acuerdo con Li y Kaiser (2011) estos profesores tendrían una mejor comprensión del conocimiento, de su estructura matemática, siendo capaces de relacionar de mejor manera estos conocimientos con ideas principales de la materia, así como con otros temas.

\section{Diseño y procedimiento}

Los datos se obtuvieron mediante la observación no participante (COHEN; MANION; MORRISON, 2018) a través de la videograbación de dos sesiones de clase consecutivas de aproximadamente 35 minutos de duración cada una. Para la videograbación se utilizaron dos cámaras: una que estaba fija (centrada en toda la clase) y otra cámara en movimiento para capturar con mayor precisión las interacciones entre los 
estudiantes y entre el profesor y los estudiantes, para así poder focalizarse en las preguntas y respuestas de las niñas y niños, así como en cómo el profesor proporciona andamiaje y guía el aprendizaje de sus estudiantes.

La experiencia de aprendizaje considerada en este estudio fue diseñada y planificada por el profesor de infantil y se fundamenta en el marco para el desarrollo del pensamiento probabilístico propuesto por Jones, Langrall, Thornton y Mogill (1997), con el fin de indagar sobre la comprensión de las primeras nociones e ideas clave asociadas al azar y la probabilidad de las niñas y niños de esta etapa educativa, tales como: espacio muestral, posibilidad de ocurrencia, probabilidad y comparación de probabilidades. Dicha experiencia de aprendizaje se divide en 2 grandes momentos que son abordados en el transcurso de las dos sesiones:

Primer momento (primera sesión): las niñas y los niños construyen y decoran conjuntamente un acuario con conchas de moluscos, algas, arena, piedras pequeñas, caracolas y peces de colores (coloreados y recortados por ellos mismos). Posteriormente discuten acerca de las características de los seres vivos que pueden vivir en el mar y que están presentes en el acuario.

Segundo momento (segunda sesión): el profesor dispone de una pecera y peces de igual forma y tamaño distribuidos como muestra la Figura 1 (5 rojos, 6 verdes y 3 amarillos).

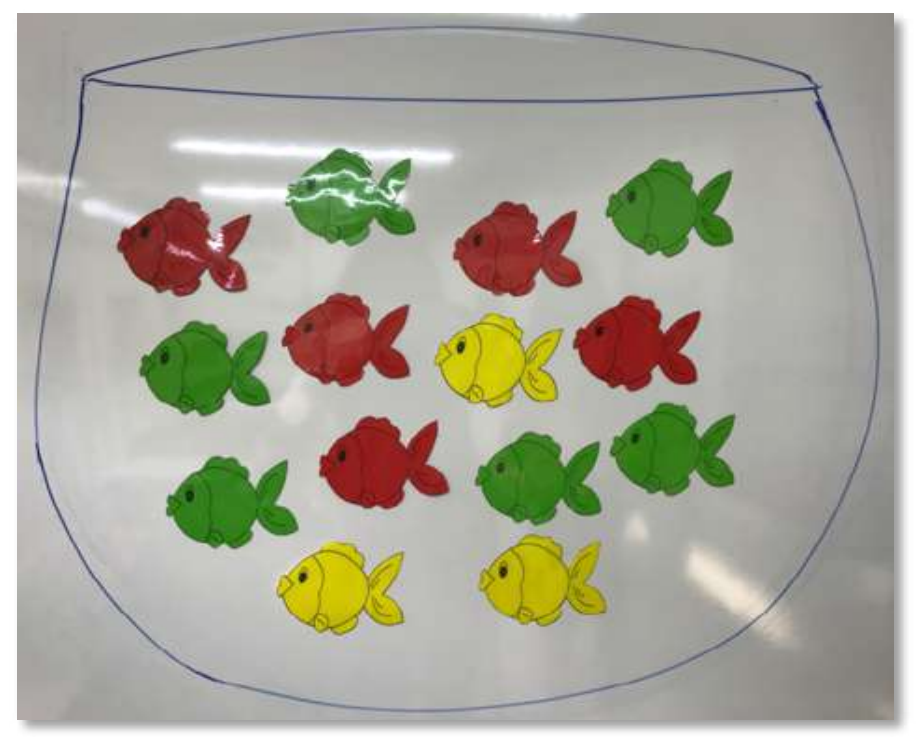

Figura 1. Apoyo visual presentado por el profesor. 
Luego, presenta una a una las siguientes preguntas (Cuadro 1), cuyo propósito es indagar en las ideas intuitivas sobre probabilidad:

Cuadro 1. Preguntas que conforman la experiencia de aprendizaje

Pregunta 1: ¿Cuántos peces hay de cada color?
Pregunta 2: ¿De qué color puede ser el pez que se saque de la pecera? ¿Por qué?
Pregunta 3: Si quiero sacar un pez con los ojos cerrados (sin mirar la pecera), ¿Qué pez es más
fácil (tiene más posibilidades) de salir?
Pregunta 4: Si quiero sacar un pez con los ojos cerrados ( sin mirar la pecera), ¿Qué pez es más
difícil (tiene menos posibilidades) de salir? ¿Por qué?
Pregunta 5: ¿Es posible sacar un pez de color anaranjado de la pecera? ¿Por qué?
Pregunta 6: Si pudiera ordenar desde el color de pez más fácil de sacar (el que tiene mayores
posibilidades de salir) al más difícil de sacar (el que tiene menos posibilidades de salir), ¿cómo
quedarían ordenados? ¿Por qué?

\section{Análisis de los datos}

Para el análisis de los datos, se aplicaron las siete fases del modelo analítico de Powell, Francisco y Maher (2003) que consisten en: a) visualización intencionada; b) descripción de los datos del video; c) identificación de sucesos críticos; d) transcripción; e) codificación; f) construcción del argumento; y g) composición narrativa. Estas fases interactivas permitieron acceder a la puesta en escena del conocimiento profesional del profesor, en especial, en cómo gestiona los procesos matemáticos a la largo de la experiencia de aprendizaje propuesta. En las tres primeras fases se observaron reiteradas veces las videograbaciones, que se segmentaron en intervalos de 5 minutos de duración, creando un registro con marcas de tiempo y breves descripciones -de carácter descriptivo y no interpretativo o inferencial- de episodios específicos (sucesos críticos) de interés para el estudio. Una vez se obtuvo una visión general de la puesta en escena de la experiencia de aprendizaje, se procedió a la fase de transcripción y codificación de los sucesos críticos con la finalidad de analizar de cerca los procesos matemáticos que el profesor gestiona a lo largo de la implementación de la experiencia de aprendizaje. En el proceso de codificación se utilizó un conjunto de descriptores asociados a cada uno de los procesos matemáticos (Cuadro 2). Lo que permitió la selección de extractos de interés (evidencia) para la construcción preliminar e inconexa del argumento de la lección. Las observaciones realizadas se aclararon a partir de los sucesos críticos, por medio de sesiones de análisis conjunto entre el investigador a cargo y el profesor de infantil. 
Cuadro 2. Descriptores utilizados en el proceso de codificación

\begin{tabular}{|c|c|}
\hline $\begin{array}{c}\text { Proceso } \\
\text { matemático }\end{array}$ & $\begin{array}{c}\text { Descriptores } \\
\text { El profesor otorga andamios que permiten a los estudiantes: }\end{array}$ \\
\hline \multirow{4}{*}{$\begin{array}{l}\text { Resolución de } \\
\text { problemas }\end{array}$} & $\begin{array}{l}\text { 1. Construir nuevos conocimientos matemáticos a través de la resolución de } \\
\text { problemas. }\end{array}$ \\
\hline & 2. Resolver problemas que surjan de las matemáticas y de otros contextos. \\
\hline & 3. Aplicar y adaptar una variedad de estrategias para resolver problemas. \\
\hline & $\begin{array}{l}\text { 4. Controlar el proceso de resolución de problemas matemáticos y reflexionar } \\
\text { sobre él. }\end{array}$ \\
\hline \multirow{4}{*}{$\begin{array}{l}\text { Razonamiento } \\
\text { y prueba }\end{array}$} & $\begin{array}{l}\text { 1. Reconocer el razonamiento y prueba como aspectos fundamentales de las } \\
\text { matemáticas. }\end{array}$ \\
\hline & 2. Formular e investigar conjeturas matemáticas. \\
\hline & 3. Desarrollar y evaluar argumentos y demostraciones matemáticas. \\
\hline & 4. Elegir y desarrollar varios tipos de razonamiento y métodos de prueba. \\
\hline \multirow{4}{*}{ Comunicación } & $\begin{array}{l}\text { 1. Organizar y consolidar su pensamiento matemático a través de la } \\
\text { comunicación. }\end{array}$ \\
\hline & $\begin{array}{l}\text { 2. Comunicar su pensamiento matemático con coherencia y claridad a los } \\
\text { compañeros, profesores y otras personas. }\end{array}$ \\
\hline & 3. Analizar y evaluar las estrategias y el pensamiento matemático de los demás. \\
\hline & $\begin{array}{l}\text { 4. Usar el lenguaje de las matemáticas para expresar ideas matemáticas con } \\
\text { precisión. }\end{array}$ \\
\hline \multirow{3}{*}{ Conexiones } & 1. Reconocer y usar conexiones entre ideas matemáticas. \\
\hline & $\begin{array}{l}\text { 2. Comprender cómo las ideas matemáticas se interconectan y construyen unas } \\
\text { sobre otras para producir un todo coherente. }\end{array}$ \\
\hline & 3. Reconocer y aplicar las matemáticas en contextos no matemáticos. \\
\hline \multirow{3}{*}{ Representación } & $\begin{array}{l}\text { 1. Crear y utilizar representaciones para organizar, registrar y comunicar ideas } \\
\text { matemáticas. }\end{array}$ \\
\hline & $\begin{array}{l}\text { 2. Seleccionar, aplicar y traducir representaciones matemáticas para resolver } \\
\text { problemas. }\end{array}$ \\
\hline & $\begin{array}{l}\text { 3. Usar representaciones para modelizar e interpretar fenómenos físicos, } \\
\text { sociales y matemáticos. }\end{array}$ \\
\hline
\end{tabular}
Fuente: NCTM (2000).

Así, a través de este proceso de análisis fue posible identificar los procesos matemáticos que surgen a lo largo de la segunda sesión de clase y describir el conocimiento profesional que manifiesta el profesor.

\section{RESULTADOS}

En este estudio, que busca analizar cómo se gestiona el desarrollo de los procesos matemáticos en el aula de Educación Infantil, se analizan las preguntas y orientaciones, otorgadas por el profesor, que llevan a los estudiantes a ampliar y/o profundizar en los conocimientos de probabilidad que se desean abordan durante el desarrollo de la clase. Para ello, a partir de la transcripción de la clase desarrollada identificamos sucesos críticos, que nos han permitido analizar las intervenciones y andamiaje que proporciona el profesor para 
conducir la enseñanza de la probabilidad en estas edades. A continuación, se describen los datos obtenidos a partir del trabajo vinculado a cada una de las seis preguntas conforman la experiencia de aprendizaje. Presentando en primer lugar un análisis referido al propósito de cada pregunta y los procesos involucrados, para luego analizar la gestión realizada por el profesor (puesta en escena) de la pregunta en cuestión.

\section{Pregunta 1 y su gestión}

En la pregunta 1 (¿cuántos peces hay de cada color?), está presente el proceso de resolución de problemas y representación. Puesto que, para dar respuesta a esta pregunta los estudiantes deberán recurrir a sus conocimientos de numeración y conteo, a través de la correspondencia uno a uno, seriación, cardinalidad y abstracción, apoyándose en la Figura presentada por el profesor, para luego adquirir ideas matemáticas nuevas y familiarizarse con las nociones del espacio muestral. Si bien esta pregunta se vincula a la resolución de problemas con apoyo en la representación, al analizar la gestión de la clase, además de estos procesos, se observa la presencia del proceso de comunicación dado que el profesor crea y utiliza representaciones para organizar y comunicar la idea matemática inicial de la clase. Un ejemplo de esto se muestra en la Figura 2.

Profesor: ya mis niños miren acá observemos la siguiente pecera, en esta pecera hay unos pececitos, ¿cierto? ¿de qué color son esos peces? (señala la pizarra y muestra el material preparado)

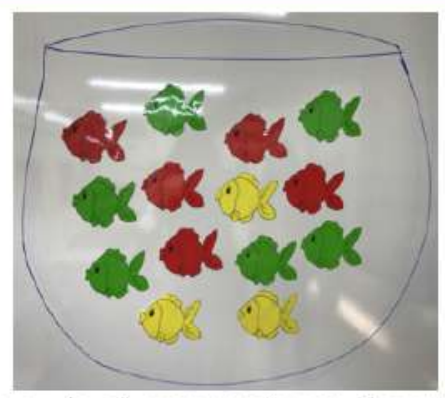

Apoyo visual presentado por el profesor.

Andrea: verdes, rojos y amarillos. Profesor: ¿Quién opina diferente a Andrea?

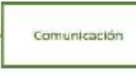

Alumnos a coro: Está bien son rojos, verdes y amarillos

Profesor: ¡Muy bien! Son rojos, verdes y amarillos. Entonces miran acá, si ahora les pido que me digan ¿cuántos peces hay de cada color?, ¿cuántos verdes?, ¿cuántos rojos? y ¿cuántos amarillos?

Figura 2. Extracto transcripción suceso crítico de la gestión de la pregunta 1. 
Otro proceso, identificado en la gestión de esta pregunta es la comunicación, puesto que el profesor constantemente invita a las niñas y los niños a compartir y aclarar sus ideas en relación con las respuestas que formulan, organizando así su pensamiento matemático y comunicándolo a los demás con coherencia y claridad. Además, a través de las preguntas que se muestran en la Figura 3, se observa que el profesor promueve que las niñas y los niños verbalicen sus resultados y pensamiento matemático asociado, favoreciendo el intercambio de ideas por medio de la interacción y puesta en común de las respuestas.

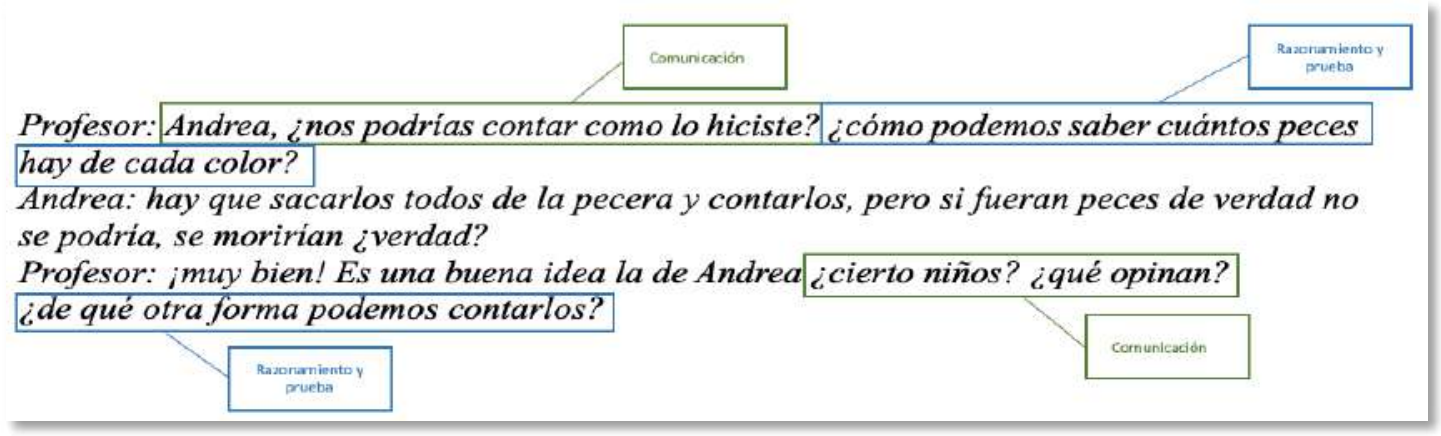

Figura 3. Extracto transcripción suceso crítico de la gestión de la pregunta 1.

Finalmente, se advierte que el proceso de razonamiento y prueba también está presente en la gestión de la pregunta 1, dado que el profesor motiva a los estudiantes a razonar respecto de las afirmaciones que realizan y los conduce a formular argumentos matemáticos con base a dichas afirmaciones.

\section{Pregunta 2 y su gestión}

En el caso de la pregunta 2 (¿de qué color puede ser el pez que se saque de la pecera? ¿Por qué?), se evidencian los procesos de resolución de problemas, y razonamiento y prueba. Dado que, por una parte, la tarea permite construir nuevos conocimientos a través de su resolución, como lo es la distinción entre sucesos. Por otra parte, para obtener la respuesta correcta las niñas y los niños deben desarrollar ideas por medio de la formulación de conjeturas matemáticas en relación con los posibles resultados que se pueden obtener al extraer un pez de la pecera, lo que les permite realizar conexiones entre las ideas matemáticas previas asociadas a las cantidades de peces de cada color y la posibilidad de ocurrencia de un determinado suceso, en este caso extraer un pez. Asimismo, la pregunta ¿Por qué?, provocará que los estudiantes usen un lenguaje matemático para expresar sus ideas y justifiquen su respuesta, beneficiando que estos comprendan que las aseveraciones 
requieren ser sustentadas o refutadas con pruebas. En este contexto, se infiere el valor que otorga el profesor al razonamiento como un aspecto fundamental en el aprendizaje de las matemáticas. Ahora, si nos situamos desde la perspectiva de la gestión de la clase y, centramos la mirada en los andamios que el profesor ofrece a los estudiantes, se observa de manera específica los procesos mencionados, en especial la resolución de problemas. Puesto que los estudiantes resuelven la tarea al distinguir entre un suceso posible y un suceso imposible, situándose desde un nuevo contexto, para avanzar hacia la construcción de nuevos conocimientos, al identificar que no se pueden extraer peces de otros colores, dado que en la pecera solo hay peces verdes, amarillos y rojos, lo que evidencia que logran distinguir entre un suceso posible y sucesos imposibles. Del mismo modo, se percibe que el proceso de razonamiento y prueba se centra en la reflexión de las respuestas otorgadas. Ejemplos de esto se muestran en la Figura 4.

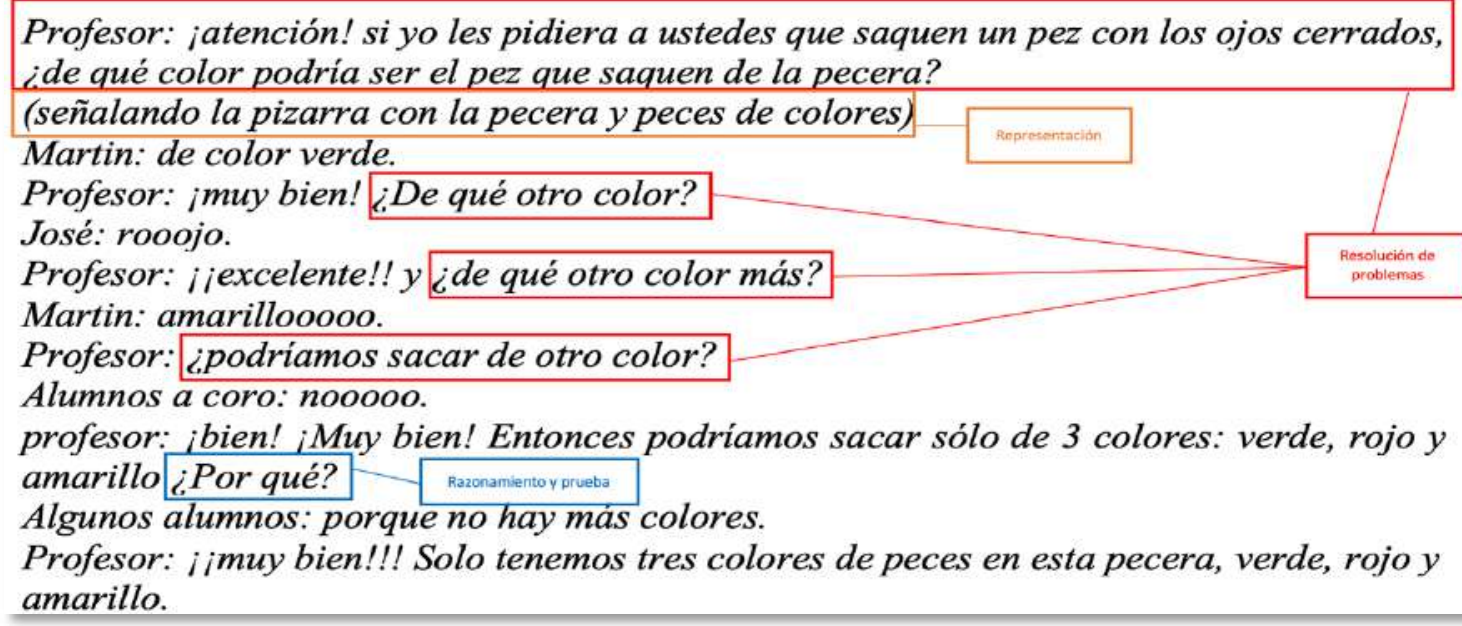

Figura 4. Extracto transcripción suceso crítico de la gestión de la pregunta 2.

Asimismo, el profesor se apoya en el proceso de representación, al señalar la pecera con peces de colores para que los estudiantes identifiquen las distintas opciones de extracción para organizar, registrar y comunicar ideas matemáticas.

\section{Pregunta 3 y su gestión}

En la pregunta 3 (si quiero sacar un pez con los ojos cerrados (sin mirar la pecera), ¿qué pez es más fácil (tiene más posibilidades) de salir?) está presente el proceso de resolución de problemas y conexión, puesto que para dar respuesta los estudiantes deberán construir nuevos conocimientos matemáticos a partir del concepto de cardinalidad, sus 
conocimientos de numeración y conteo vinculándoles al grado de posibilidad de ocurrencia de un evento. Al analizar la gestión de la clase, podemos evidenciar cómo el profesor conduce el proceso de resolución de problemas y además fomenta el proceso de conexión al proponer preguntas, tales como, ¿hay alguna diferencia entre sacar un pez rojo, verde o amarillo? ¿cuántos peces tenemos de cada color? Puesto que, estas interrogantes promueven el reconocimiento y uso de conexiones entre distintas ideas matemáticas como, por ejemplo, distinguir los casos favorables asociados a la posibilidad de ocurrencia de un evento y comparar cantidades. Otro proceso que emerge de la gestión de la clase es la comunicación, por medio del uso de representaciones generado por el apoyo visual que presenta el profesor en el pizarrón para organizar, registrar y comunicar las ideas matemáticas involucradas. Del mismo modo, el discutir acerca del grado de posibilidad de ocurrencia es objeto de reflexión para los estudiantes, suscitando la organización y consolidación del pensamiento matemático a través de la comunicación, ya sea de manera oral o escrita. Un ejemplo de ello se observa en la Figura 5.

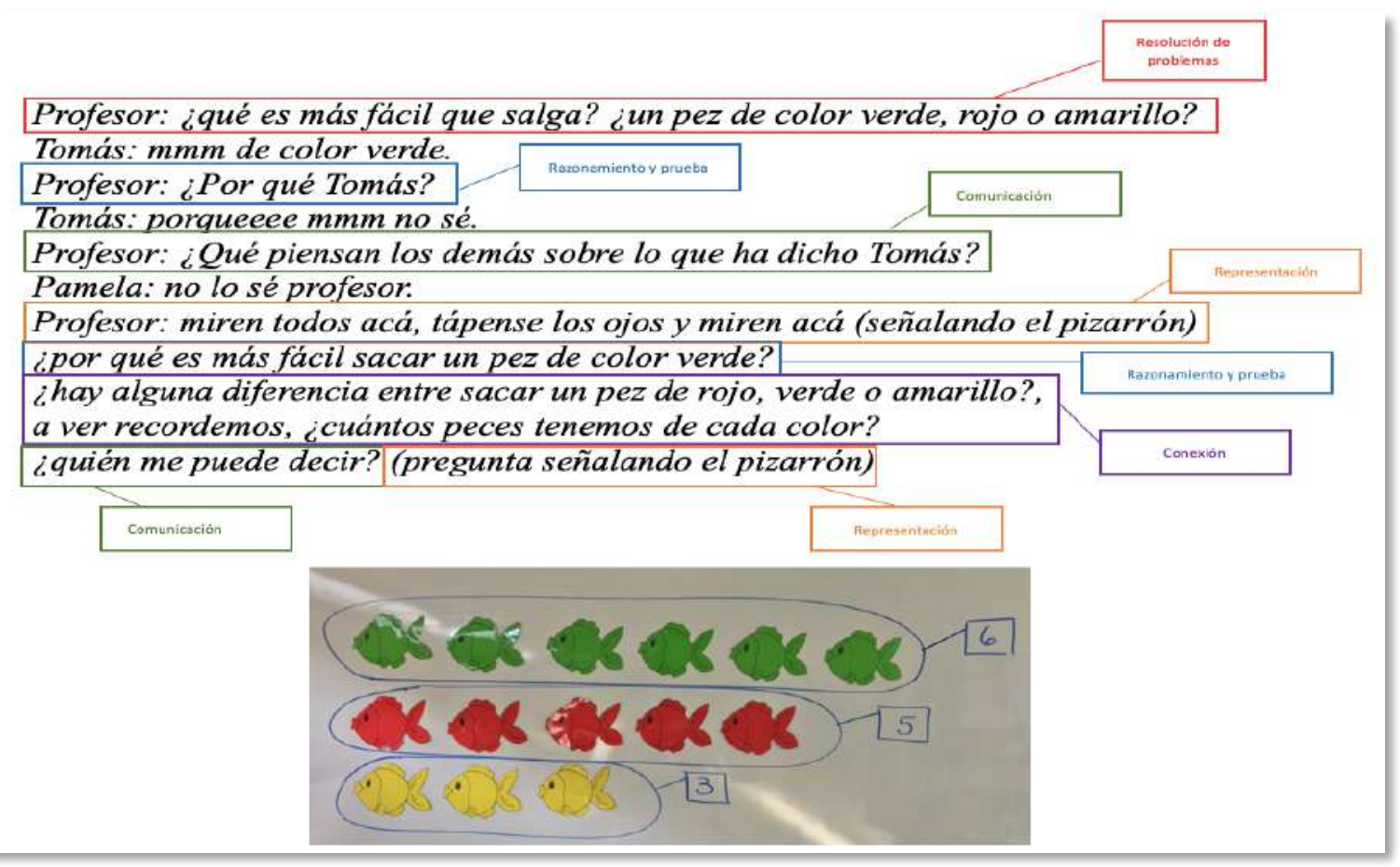

Figura 5. Extracto transcripción suceso crítico de la gestión de la pregunta 3.

Por último, durante la gestión de la clase, se evidencia cómo el profesor plantea preguntas orientadoras que suscitan el proceso de razonamiento y prueba, ayudando a los estudiantes a comprender las afirmaciones que realizan como, por ejemplo, ¿por qué Tomás? 
¿por qué es más fácil sacar un pez de color verde?

\section{Pregunta 4 y su gestión}

En relación con la pregunta 4 (si quiero sacar un pez con los ojos cerrados (sin mirar la pecera), ¿qué pez es más difícil (tiene menos posibilidades) de salir? ¿Por qué?) están presentes los procesos de resolución de problemas, conexión y, razonamiento y prueba. Dado que, para responder a tales preguntas, los estudiantes deberán, al igual que en la pregunta 3, construir nuevos conocimientos matemáticos vinculados al grado de posibilidad de ocurrencia de un evento. A su vez, los estudiantes deberán reconocer y usar conexiones entre ideas matemáticas para distinguir los casos favorables asociados a la posibilidad de ocurrencia de un evento, y de esta forma justificar su razonamiento.

Desde la perspectiva de la gestión de la clase, en el primer momento de la interacción es posible evidenciar, de manera explícita, los procesos de resolución de problemas y, razonamiento y prueba, como se aprecia en la Figura 6. Sin embargo, se advierte que el profesor no conduce el proceso de conexión.

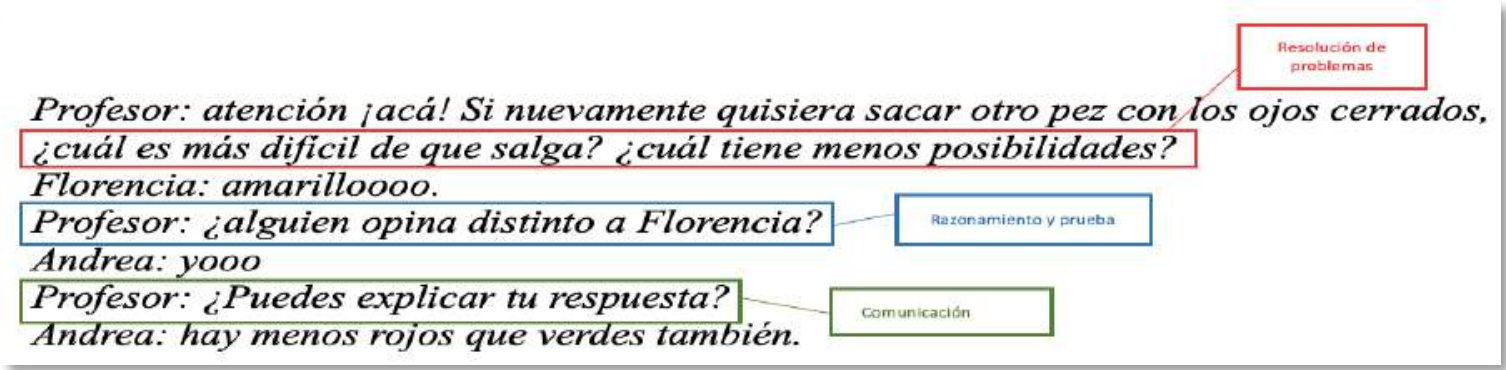

Figura 6. Extracto transcripción suceso crítico de la gestión de la pregunta 4.

Por otra parte, durante la gestión de la clase es posible observar cómo emerge el proceso de comunicación, puesto que el profesor invita constantemente a los estudiantes a expresar su pensamiento matemático de manera clara y coherente a los compañeros a través del intercambio de ideas.

\section{Pregunta 5 y su gestión}

En el caso de la pregunta 5 (¿es posible sacar un pez de color anaranjado de la pecera? ¿Por qué?) están presentes los procesos de resolución de problemas, conexión, razonamiento y prueba. Dado que, para dar respuesta a las interrogantes los estudiantes deberán construir nuevos los conocimientos matemáticos conectados con sus conocimientos previos, lo que les permitirá distinguir entre sucesos posibles o imposibles. 
Asimismo, los estudiantes deberán formular conjeturas y reflexionar acerca del grado de posibilidad de ocurrencia. Respecto de la gestión de la clase, se evidencia de manera clara cómo el profesor conduce el proceso de resolución de problemas, al inicio de la interacción (Figura 7). No obstante, se advierte que este no suscita el proceso de razonamiento y prueba ni tampoco el de conexión.

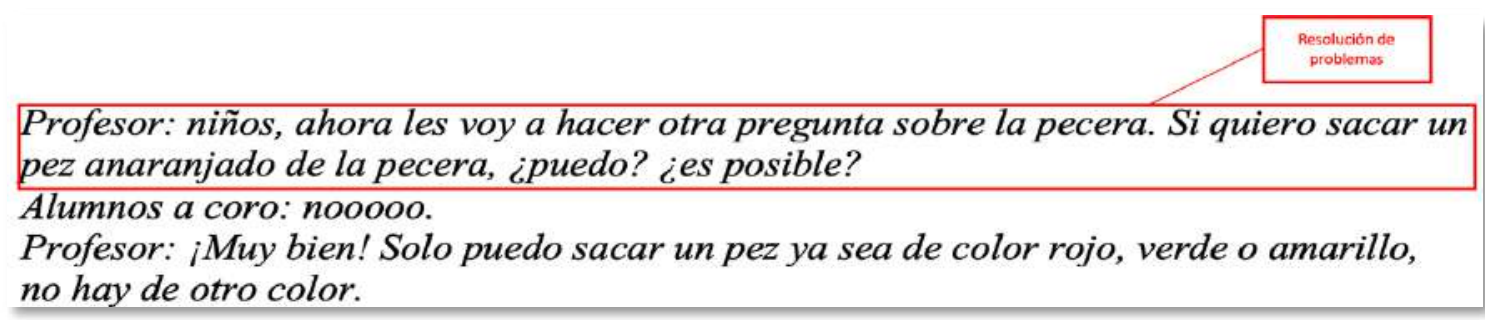

Figura 7. Extracto transcripción suceso crítico de la gestión de la pregunta 5.

\section{Pregunta 6 y su gestión}

En la pregunta 6 (si pudiera ordenar desde el color de pez más fácil de sacar (el que tiene mayores posibilidades de salir) al más difícil de sacar (el que tiene menos posibilidades de salir), ¿cómo quedarían ordenados? ¿Por qué?) están presentes los procesos de resolución de problemas, conexión y, razonamiento y prueba. Puesto que, los estudiantes para dar respuesta deberán recurrir a sus conocimientos de cantidad y clasificación de elementos, a través de la organización y registro del color de cada pez, para luego adquirir nociones matemáticas nuevas y familiarizarse con los sucesos posibles o no posibles. Igualmente, los estudiantes deberán interpretar el experimento y reflexionar acerca de su grado de posibilidad de ocurrencia. Si nos situamos desde la perspectiva de la gestión de la clase, es posible evidenciar el proceso de resolución de problemas y conexión, sin embargo, no se observa que el profesor conduzca el proceso de razonamiento y prueba. Por otro lado, a partir de la gestión de la clase emerge el proceso de comunicación, puesto que el profesor invita a los estudiantes a interpretar las ideas de otros compañeros y expresar su pensamiento matemático con coherencia y claridad. Otro proceso que aflora en la gestión de la clase es la representación, al utilizar el pizarrón para interpretar la tarea, organizando y registrando los peces de colores para apoyar el proceso de conexión. Un extracto de este suceso crítico se muestra en la Figura 8. 


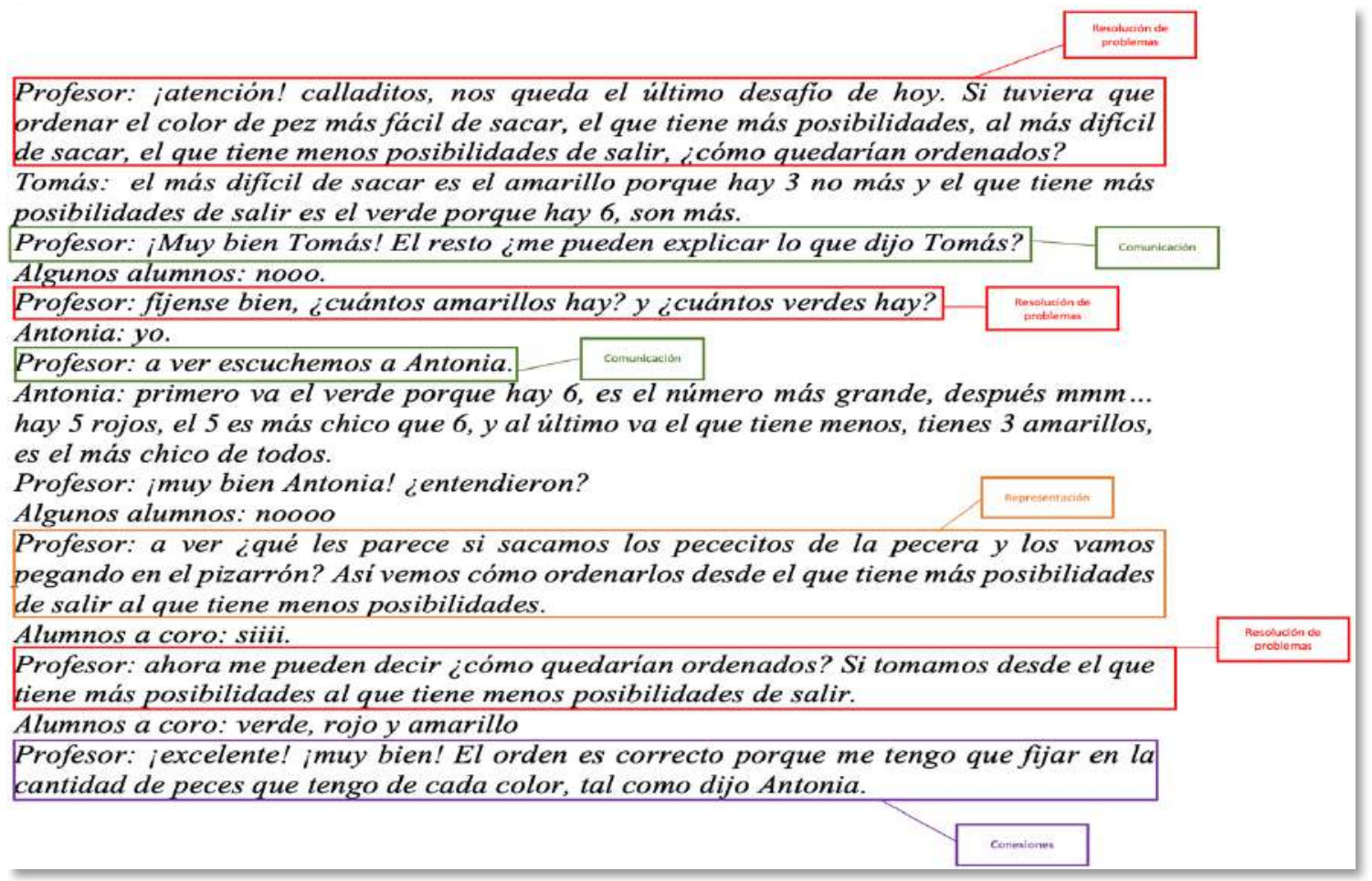

Figura 8. Extracto transcripción suceso crítico de la gestión de la pregunta 6.

Así, a partir del análisis de la experiencia de aprendizaje diseñada por el profesor de infantil, hemos identificado los procesos matemáticos presentes en cada una de las preguntas planteadas a las niñas y los niños, que se resumen en la Tabla 1. Es importante tener en cuenta que una misma tarea puede atender a uno o más de los procesos matemáticos.

Tabla 1. Procesos matemáticos identificados en el diseño

\begin{tabular}{|c|c|c|c|c|c|}
\hline \multirow{3}{*}{ Preguntas } & \multicolumn{5}{|c|}{ Procesos matemáticos identificados } \\
\cline { 2 - 6 } & $\begin{array}{c}\text { Resolución } \\
\text { de problemas }\end{array}$ & $\begin{array}{c}\text { Razonamiento } \\
\text { y prueba }\end{array}$ & Comunicación & Conexiones & Representación \\
\hline 1 & $\mathrm{x}$ & & & & $\mathrm{X}$ \\
\hline 2 & $\mathrm{x}$ & $\mathrm{x}$ & & $\mathrm{x}$ & \\
\hline 3 & $\mathrm{x}$ & $\mathrm{x}$ & & $\mathrm{x}$ & \\
\hline 4 & $\mathrm{x}$ & $\mathrm{x}$ & & $\mathrm{x}$ & \\
\hline 5 & $\mathrm{x}$ & $\mathrm{x}$ & & $\mathrm{x}$ & \\
\hline 6 & $\mathrm{x}$ & $\mathrm{x}$ & & & \\
\hline
\end{tabular}

Como se observa en la Tabla 1, existe un predominio de la resolución de problemas, seguido de razonamiento y prueba, conexiones y representación. Cabe señalar que estos procesos matemáticos corresponden a los identificados exclusivamente en la experiencia de aprendizaje, previo a su implementación. Ahora bien, al analizar la implementación de 
la experiencia de aprendizaje, específicamente la gestión de las preguntas planteadas, se evidencian procesos matemáticos que no eran explícitos en el diseño de la experiencia de aprendizaje (Tabla 2).

Tabla 2. Procesos matemáticos identificados en la gestión

\begin{tabular}{|c|c|c|c|c|c|}
\hline \multirow{2}{*}{$\begin{array}{l}\text { Gestión de } \\
\text { la pregunta }\end{array}$} & \multicolumn{5}{|c|}{ Procesos matemáticos identificados } \\
\hline & $\begin{array}{l}\text { Resolución } \\
\text { de problemas }\end{array}$ & $\begin{array}{c}\text { Razonamiento } \\
\text { y prueba }\end{array}$ & Comunicación & Conexiones & Representación \\
\hline 1 & $\mathrm{x}$ & $\mathrm{x}$ & $\mathrm{x}$ & & $\mathrm{x}$ \\
\hline 2 & $\mathrm{x}$ & $\mathrm{x}$ & & & $\mathrm{x}$ \\
\hline 3 & $\mathrm{x}$ & $\mathrm{x}$ & $\mathrm{X}$ & $\mathrm{X}$ & $\mathrm{x}$ \\
\hline 4 & $\mathrm{x}$ & $\mathrm{x}$ & $\mathrm{x}$ & & \\
\hline 5 & $\mathrm{x}$ & & & & \\
\hline 6 & $\mathrm{x}$ & & $x$ & $\mathrm{x}$ & $\mathrm{X}$ \\
\hline
\end{tabular}

Lo anterior, no hace más que reflejar la riqueza de analizar los conocimientos que el profesorado pone en juego al enseñar probabilidad, lo que aporta evidencias, en nuestro caso, de los procesos matemáticos que el profesor moviliza al enseñar probabilidad en el aula de Educación Infantil.

\section{CONSIDERACIONES FINALES}

En las últimas décadas se observa un creciente interés por el desarrollo de investigaciones en torno a la formación y desarrollo del profesorado sobre todo en el área de matemáticas (e.g. HILL; BALL; SCHILLING, 2008; EVEN; BALL, 2009). Esto producto de la necesidad de contar con profesores mejor preparados para la enseñanza de esta disciplina, sobre todo en los primeros niveles educativos, pues "los profesores son la clave de oportunidad de aprendizaje de las matemáticas" (EVEN; BALL, 2009, p.1-2), por lo que finalmente la calidad de la enseñanza depende de ellos, de su conocimiento y su preparación para enseñar, la cual impacta directamente en el aprendizaje y desarrollo de competencias matemáticas de los estudiantes (DARLING-HAMMOND; WEI; JOHNSON, 2009). Por tanto, si se desea mejorar la formación matemática de los estudiantes, es necesario prestar especial atención al conocimiento profesional del profesor, entendido este como "el conjunto de todos los saberes y experiencias que un profesor posee y de los que hace uso en el desarrollo de su labor docente, que va construyendo desde su formación inicial y durante toda su carrera profesional” (CLIMENT, 2002, p.52-53). Específicamente, surge la necesidad de focalizarse en alcanzar una comprensión en profundidad de las 
características y naturaleza del conocimiento matemático necesario para enseñar. Para ello, resulta de gran interés indagar en un campo aún poco estudiado y que reclama atención por parte de la Didáctica de la Matemática: el conocimiento matemático para la enseñanza que ponen en juego los profesores en ejercicio en el aula, en sus prácticas de enseñanza, al enseñar un determinado tema matemático. Es en este contexto, que en este estudio hemos querido avanzar en este sentido, por lo que nos focalizamos en el análisis del conocimiento del profesorado de Educación Infantil para enseñar probabilidad, poniendo especial atención en los procesos matemáticos. Pues no basta con que un profesor posea un alto conocimiento del contenido matemático a enseñar, sino también debe contar con un amplio conocimiento sobre cómo dicho conocimiento se adquiere y de cómo ese conocimiento se utiliza en contextos diversos, es decir, debe conocer en profundidad los procesos matemáticos (NCTM, 2000).

Nuestros resultados evidencian la presencia de los procesos matemáticos de resolución de problemas, razonamiento y prueba, comunicación, conexiones y representación con distintos niveles de profundidad y de atención en los diferentes sucesos críticos de la sesión de clase analizada. Donde la representación y la comunicación constituye un elemento clave al momento de promover la resolución de problemas, el razonamiento y prueba y las conexiones. Lo que confirma, que las niñas y los niños de estas edades necesitan iniciar el aprendizaje de las primeras nociones probabilísticas en contextos de enseñanza que permitan visualizar las ideas matemáticas de manera concreta, tanto a partir de objetos de la vida cotidiana como de materiales manipulativos, y de la acción directa sobre estos objetos (ALSINA, 2017; ALSINA; VÁSQUEZ, 2016; VÁSQUEZ, 2014; VÁSQUEZ; ALSINA, 2019). No cabe duda, que junto al diseño de experiencias de aprendizajes matemáticamente competenciales, un aspecto clave es la gestión que el profesorado realiza de dicha actividades, lo que puede provocar que emerjan y se movilicen procesos matemáticos que no son del todo evidentes en el diseño de la experiencia de aprendizaje y que se hacen visibles a partir de la formulación de buenas preguntas que invitan a las niñas y niños a razonar, comunicar, argumentar y justificar sus acciones e ideas matemáticas en torno a la probabilidad; en un ambiente de comunicación matemática en el aula, en el que la interacción, la negociación, el diálogo y el uso de lenguaje probabilístico cada vez más preciso se hacen presentes. Se trata, pues, de 
promover desde temprana edad una enseñanza de la estadística y la probabilidad a través de los procesos matemáticos (NCTM, 2000; ALSINA, 2013, 2017; VÁSQUEZ; ALSINA, 2019; VÁSQUEZ; CORONATA; RIVAS, en prensa), que permita a las niñas y los niños dar respuesta a los requerimientos del mundo actual, así como contar con un pensamiento crítico y conocimientos que les permitan allanar el camino para que a futuro puedan interpretar información proveniente de diversas fuentes, con el fin de tomar decisiones de manera informada, o bien, simplemente distinguir entre aquella información que no es relevante o que no se ha comunicado adecuadamente. Por otra parte, las limitaciones de la investigación tienen relación con el diseño de la misma, puesto que corresponde a un estudio de caso, lo que no permite realizar generalizaciones respecto de los procesos matemáticos que se movilizan al enseñar probabilidad en el aula de Educación Infantil.Por último, esta investigación permite abrir futuras líneas de indagación ampliando el estudio a otros profesores. Estos resultados pueden ser relevantes dado que se pueden utilizar de base para el diseño de experiencias de aprendizaje que permitan promover los procesos matemáticos en el aula de Educación Infantil.

\section{Agradecimientos}

Trabajo realizado en el marco del proyecto FONDECYT No 11150412 y Beca de doctorado en el extranjero $N^{\circ} 72200447$ financiado por la Comisión Nacional de Investigación Científica y Tecnológica de Chile.

\section{REFERENCIAS}

ALSINA, Á. La estadística y la probabilidad en Educación Infantil: conocimientos disciplinares, didácticos y experienciales. Didácticas Específicas, n. 7, p. 4-22, 2013.

ALSINA, Á. Procesos matemáticos en Educación Infantil: 50 ideas clave. Números, n. 86, p. 5-28, 2014.

ALSINA, Á. Contextos y propuestas para la enseñanza de la estadística y la probabilidad en Educación Infantil: un itinerario didáctico. Revista Épsilon, v. 34, n. 95, p. 25-48, 2017.

ALSINA, Á. Itinerarios didácticos para la enseñanza de las matemáticas (6-12 años). Barcelona: Editorial Graó, 2019.

ALSINA, Á.; VÁSQUEZ, C. De la competencia matemática a la alfabetización probabilística en el aula: elementos para su caracterización y desarrollo. UNION, Revista Iberoamericana de Educación Matemática, n. 48, p. 41-58, 2016. 
CARPENTER, T.P.; LEVI, L. Developing conceptions of algebraic reasoning in the primary grades. Madison: University of Wisconsin-Madison, 1999.

CLIMENT, N. El desarrollo profesional del maestro de primaria respecto de la enseñanza de la matemática: Un estudio de caso. Tesis doctoral - Universidad de Huelva, Huelva, 2002.

COHEN, L.; MANION, L.; MORRISON, K. Research methods in education (Octava edición). Nueva York, NY, Routledge, 2018.

DARLING-HAMMOND, L.; WEI, R. C.; JOHNSON, C. M. Teacher Preparation and Teacher Learning: A Changing Policy Landscape. In: Sykes, G; Schneider, B. L.; Plank, D. N. (Eds.), Handbook of Education Policy Research. New York: American Educational Research Association and Routledge, 2009. p. 613- 636.

EVEN, R.; BALL, D. L. The professional education and development of teachers of mathematics - the 15th ICMI Study. New York, NY: Springer, 2009.

HERNÁNDEZ, R.; FERNÁNDEZ, C.; BAPTISTA, P. Metodología de la investigación. 5. ed. México: McGraw Hill, 2010.

HILL, H.C.; BALL, D.L.; SCHILLING, S.G. Unpacking pedagogical content knowledge: Conceptualizing and measuring teachers topic specific knowledge of students. Journal for Research in Mathematics Education, v. 39, n. 4, p. 372-400, 2008.

JONES, G.; LANGRALL, C.; THORNTON, C.; MOGILL, T. A framework for assessing and nurturing young children's thinking in probability. Educational Studies in Mathematics, n. 32, p. 101-125, 1997.

LI, Y. Y KAISER, G. Expertise in mathematics instruction: Advancing research and practice from an international perspective. In: Li, Y.; Kaiser, G. (Eds.). Expertise in mathematics instruction: An international perspective. New York: Springer, 2011. p. $3-15$.

NATIONAL ASSOCIATION FOR THE EDUCATION OF YOUNG CHILDREN; NATIONAL COUNCIL OF TEACHERS OF MATHEMATICS. Matemáticas en la educación infantil: Facilitando un buen inicio. Declaración conjunta de posición. Edma 06: Educación Matemática en la Infancia, v. 2, n. 1, p. 1-23, 2013.

NCTM. Principles and Standards for School Mathematics. Reston: The National Council of Teachers of Mathematics, 2000.

OCDE. Future of Education and Skills 2030: OECD Learning Compass 2030. Paris: OECD, 2019. Recuperado de http://www.oecd.org/education/2030-project/teaching-andlearning/learning/core-foundations/Core_Foundations_for_2030_concept_note.pdf

PASSOLUNGHI, M. C.; LANFRANCHI, S. Domain-specific and domain-general precursors of mathematical achievement: A longitudinal study from kindergarten to first grade. British Journal of Educational Psychology, v. 82, n. 1, p. 42-63, 2012.

POWELL, A. B.; FRANCISCO, J. M.; MAHER, C. A. An analytical model for studying the development of learners' mathematical ideas and reasoning using videotape data. Journal of Mathematical Behavior, v. 22, n. 4, p. 405-435, 2003. https://doi.org/10.1016/j.jmathb.2003.09.002. 
ROJAS, N.; CARRILLO, J.; FLORES, P. Características para identificar a profesores de matemáticas expertos. En: Estepa, A.; Contreras, A.; Deulofeu, J.; Penalva, M. C.; García, F. J; Ordoñez, L. (Eds.), Investigación en Educación Matemática XVI. Jaén: SEIEM, 2012. p. $479-485$.

STAKE, R. Investigación con estudio de casos. Madrid: Morata, S.L, 2020.

VÁSQUEZ, C. Evaluación de los conocimientos didáctico-matemáticos para la enseñanza de la probabilidad de los profesores de Educación Primaria en activo. Tesis Doctoral - Universidad de Girona, Girona, España, 2014.

VÁSQUEZ, C. Surgimiento del Lenguaje Probabilístico en el Aula de Educación Primaria. REnCiMa, v. 9, n. 2, p. 374-389, 2018.

VÁSQUEZ, C. Educación estocástica: una herramienta para formar ciudadanos de sostenibilidad. Revista Matemática, Educación y Sociedad, v. 3, n. 2, p. 1-20, 2020.

VÁSQUEZ, C.; ALSINA, Á. Lenguaje probabilístico: un camino para el desarrollo de la alfabetización probabilística. Un estudio de caso en el aula de Educación Primaria. Bolema, v. 31, n. 57, p. 454-478, 2017. http://dx.doi.org/10.1590/1980-4415v31n57a22

VÁSQUEZ, C.; ALSINA, Á. Intuitive ideas about chance and probability in children from 4 to 6 years old. Revista Acta Scientiae, v. 21, n. 3, p. 131-154, 2019. DOI: 10.17648/acta.scientiae.v21 iss3id5215

VÁSQUEZ, C.; CABRERA, G. (en revisión). La enseñanza de estadística y probabilidad en Educación Infantil y Primaria: reflexiones desde una perspectiva internacional.

VÁSQUEZ, C.; ALVARADO, H.; RUZ, F. Actitudes de futuras maestras de Educación Infantil hacia la estadística, la probabilidad y su enseñanza. Revista Educación Matemática, v. 31, n. 3, p. 177-202, 2019. DOI: 10.24844/EM3103.0

VÁSQUEZ, C.; CORONATA, C.; RIVAS, H. (en prensa). Enseñanza de la estadística y la probabilidad de los 4 a los 8 años de edad: una aproximación desde los procesos matemáticos en los libros de texto chilenos.

VÁSQUEZ, C.; DÍAZ-LEVICOY, D., CORONATA, C.; ALSINA, Á. Alfabetización estadística y probabilística: primeros pasos para su desarrollo desde la Educación Infantil. Cadernos Cenpec, v. 8, n. 1, p. 54-179, 2018.

VÁSQUEZ, C.; DÍAZ-LEVICOY, D.; ARTEAGA, P. Objetos matemáticos ligados a la estadística y la probabilidad en Educación Infantil: un análisis desde los libros de texto. Bolema, v. 34, n. 67, p. 480-500, 2020.

YUROVSKY, D.; BOYER, T.; SMITH, L. B.; YU, C. Probabilistic cue combination: Less is more. Developmental Science, v. 16, n. 2, p. 149-158, 2013.

Submetido em 15 de março de 2021. Aprovado em 30 de abril de 2021. 